\title{
THE METRIC APPROXIMATION PROPERTY IN NON-ARCHIMEDEAN NORMED SPACES
}

\author{
Cristina Perez-Garcia and Wilhelmus H. Schikhof \\ Universidad de Cantabria, Spain and Nijmegen, The Netherlands \\ This paper is dedicated to the second author, who passed away on May 1, 2014.
}

\begin{abstract}
A normed space $E$ over a rank 1 non-archimedean valued field $K$ has the metric approximation property (MAP) if the identity on $E$ can be approximated pointwise by finite rank operators of norm 1 .

Characterizations and hereditary properties of the MAP are obtained. For Banach spaces $E$ of countable type the following main result is derived: $E$ has the MAP if and only if $E$ is the orthogonal direct sum of finitedimensional spaces (Theorem 4.9). Examples of the MAP are also given. Among them, Example 3.3 provides a solution to the following problem, posed by the first author in $[8,4.5]$. Does every Banach space of countable type over $K$ have the MAP?
\end{abstract}

\section{INTRODUCTION}

The study of Grothendieck's approximation in non-archimedean Banach spaces was initiated in [8]. In the present paper we derive new results leading to improvements of [8] (see e.g. Theorem 3.2). Also, we give (Example 3.3) a negative answer to the following problem, posed in $[8,4.5]$. Does every Banach space of countable type over $K$ have the MAP? As an application of Example 3.3 we additionally prove that the problem raised in $[9$, p. 95$]$ has an affirmative answer, even for locally convex spaces of countable type.

In Section 5 we compare the results given in this paper with their classical versions, for Banach spaces over the real or complex field. This comparison, together with the one carried out in [8, Section 6$]$, reveals sharp and interesting contrasts between the classical MAP and its non-archimedean counterpart.

2010 Mathematics Subject Classification. 46S10, 46B28.

Key words and phrases. Non-archimedean normed spaces, pseudoreflexivity, metric approximation property, finite-dimensional decomposition. 
For explanation of terminology and symbols, see Section 2.

We recall the following fundamental notion from [8], where, for convenience (see Theorem 4.4 and Corollary 4.5), we include also non-complete spaces.

Definition 1.1. Let $\lambda \in \mathbb{R}, \lambda \geq 1$. A normed space $E$ over $K$ has the $\lambda$-bounded approximation property $(\lambda$-BAP) if for each $\varepsilon>0$ and each finite set $X \subset E$ there is a finite rank operator $T: E \rightarrow E$ with $\|T\| \leq \lambda$ and $\|T(x)-x\| \leq \varepsilon$ for all $x \in X$. E is said to have the metric approximation property (MAP) if it has the 1-BAP.

\section{Preliminaries}

By "classical theory" we mean functional analysis over $\mathbb{R}$ or $\mathbb{C}$.

Throughout $K:=(K,||$.$) is a non-archimedean non-trivially valued field$ that is complete with respect to the metric induced by the valuation $|$.$| :$ $K \rightarrow[0, \infty)$.

For basics on valued fields, see $[1,10,11,13]$. For background on nonarchimedean functional analysis, see $[9,12,13]$.

From now on in this paper $E, F$ are non-archimedean normed spaces (over $K$ ).

For convenience we recall the following.

For a set $X \subset E,[X]$ denotes the linear hull of $X$. If $\left(D_{i}\right)_{i \in I}$ is a family of subspaces of $E$, then the linear hull of $\bigcup_{i} D_{i}$ is denoted by $\sum_{i} D_{i}$.

By $L(E, F)$ we mean the $K$-vector space of all continuous linear maps (or operators) $T: E \rightarrow F$ with the norm $T \mapsto\|T\|:=\min \{M \geq 0$ : $\|T(x)\| \leq M\|x\|$ for all $x \in E\}$. If $F$ is a Banach space then so is $L(E, F)$. If $T \in L(E, F)$ and $D$ is a subspace of $E$, by $T \mid D$ we denote the restriction of $T$ to $D$. We write $E^{\prime}:=L(E, K), L(E):=L(E, E)$. By $I_{E}$ we mean the identity $E \rightarrow E$. Also, $F R(E, F):=\{T \in L(E, F): \operatorname{dim} T(E)<\infty\}$, is the space of the finite rank operators $E \rightarrow F$. We put $F R(E):=F R(E, E)$.

$E$ is called pseudoreflexive ([13, p. 60]) if the canonical operator $j_{E}$ : $E \rightarrow E^{\prime \prime}$ defined by $j_{E}(x)(f):=f(x)\left(x \in E, f \in E^{\prime}\right)$ is isometric, i.e. if (for $E \neq\{0\})\|x\|=\sup \left\{|f(x)| /\|f\|: f \in E^{\prime}, f \neq 0\right\}$ for all $x \in E$. If the valuation of $K$ is dense, $E$ is pseudoreflexive if and only if $E$ is normpolar, i.e. $\|x\|=\sup \left\{|f(x)|: f \in E^{\prime},\|f\| \leq 1\right\}$ for all $x \in E$. If $K$ is spherically complete every space $E$ is pseudoreflexive $([13,4.35])$. But if $K$ is not spherically complete the space $\ell^{\infty} / c_{0}$ is not pseudoreflexive; in fact, $\left(\ell^{\infty} / c_{0}\right)^{\prime}=\{0\}([13,4.3])$.

Two subspaces $D_{1}, D_{2}$ of $E$ are called orthogonal (notation $D_{1} \perp D_{2}$ ) if $\left\|d_{1}+d_{2}\right\|=\max \left(\left\|d_{1}\right\|,\left\|d_{2}\right\|\right)$ for all $d_{1} \in D_{1}, d_{2} \in D_{2}$. If, in addition, $D_{1}+D_{2}$ $=E$ we say that $D_{1}$ and $D_{2}$ are each other's orthocomplement. For $x, y \in E$ we sometimes write $x \perp y$ in place of $K x \perp K y$ and say that $x$ and $y$ are orthogonal. By $[13,3.2]$ this holds if and only if $\|\mu x+y\| \geq\|\mu x\|$ (or $\geq\|y\|$ ) for all $\mu \in K$. 
An operator $P \in L(E)$ is called a projection if $P^{2}=P$, an orthoprojection if, in addition, $\operatorname{Ker} P \perp P(E)$ (which is equivalent to $\|P\| \leq 1$ ).

A system $\left(D_{i}\right)_{i \in I}$ of subspaces of $E$ is called (an) orthogonal (system) if $D_{i} \perp \sum_{j \neq i} D_{j}$ for all $i \in I$. Analogously, a collection $\left(x_{i}\right)_{i \in I}$ of vectors in $E$ is called orthogonal if $\left(K x_{i}\right)_{i \in I}$ is orthogonal. If, in addition, $\left\|x_{i}\right\|=1$ for all $i \in I$, it is called orthonormal. An orthogonal system $\left(x_{i}\right)_{i \in I} \subset E \backslash\{0\}$ is called an orthogonal base (of $E$ ) if each $x \in E$ has a (unique) expansion $x=\sum_{i} \lambda_{i} x_{i}$, where $\lambda_{i} \in K$ for all $i$. In the same spirit we have the notion of an orthonormal base. For example, in the Banach space $c_{0}$ of all null sequences in $K$ (with the maximum norm), the unit vectors form an orthonormal base.

We also will need the following extension of the notion of orthogonality. Let $0<t \leq 1$. A system $\left(x_{i}\right)_{i \in I}$ of vectors in $E$ is called $t$-orthogonal if $\left\|\sum_{j \in J} \lambda_{j} x_{j}\right\| \geq t \max _{j \in J}\left\|\lambda_{j} x_{j}\right\|$ for all finite sets $J \subset I$ and $\lambda_{j} \in K(j \in J)$. A $t$-orthogonal system $\left(x_{i}\right)_{i \in I} \subset E \backslash\{0\}$ is called a t-orthogonal base (of $E$ ) if each $x \in E$ has a (unique) expansion $x=\sum_{i} \lambda_{i} x_{i}$, where $\lambda_{i} \in K$ for all $i$. Notice that 1-orthogonal systems and bases are nothing but orthogonal systems and bases.

$E$ is said to be of countable type if there is a countable set in $E$ whose linear hull is dense. We quote the following result.

Theorem 2.1. ([9, 2.3.7, 2.3.25]) A space of countable type has, for each $t \in(0,1)$, a t-orthogonal base. It has an orthogonal base if $K$ is spherically complete.

Let $\left(E_{i}\right)_{i \in I}$ be a system of normed spaces. Its orthogonal direct sum $\bigoplus_{i} E_{i}$ is the space of all $\left(x_{i}\right)_{i \in I} \in \prod_{i} E_{i}$ for which $\lim _{i}\left\|x_{i}\right\|=0$, normed by $\left(x_{i}\right)_{i \in I} \longmapsto \max _{i}\left\|x_{i}\right\|$. The subspace of all $\left(x_{i}\right)_{i \in I} \in \bigoplus_{i} E_{i}$ for which $\left\{i \in I: x_{i} \neq 0\right\}$ is finite, is called the algebraic orthogonal direct sum $\bigoplus_{i}^{a} E_{i}$. It is a dense subspace of $\bigoplus_{i} E_{i}$. If each $E_{i}$ is a Banach space then so is $\bigoplus_{i} E_{i}$.

In classical Grothendieck's approximation theory the notion of the finitedimensional decomposition property plays a role (see e.g. $[2,6.1]$ ). In our theory we modify this concept as follows.

A Banach space $E$ has the finite-dimensional decomposition property (FDDP) if it is the orthogonal direct sum of a system of finite-dimensional spaces. If $K$ is spherically complete, every finite-dimensional space has an orthogonal base (Theorem 2.1), so $E$ has the FDDP if and only if $E$ has an orthogonal base. However, if $K$ is not spherically complete there exist various kinds of finite-dimensional spaces without orthogonal base (see [6]); for these $K$ the class of Banach spaces with the FDDP can be viewed as a natural proper generalization of the class of Banach spaces with an orthogonal base.

\section{Examples AND CharaCterizations OF the MAP}

It was shown in [8] that a large amount of non-archimedean normed spaces have the $\lambda$-BAP $(\lambda>1)$ and the MAP. In fact, the following result holds. 
THEOREM 3.1 ([8, 3.3]).

(i) Every norm-polar space $E$ has the $\lambda$-BAP for all $\lambda>1$.

(ii) Suppose either $K$ is spherically complete or $E$ has an orthogonal base. Then $E$ has the MAP.

For examples of Banach spaces (e.g. valued field extensions; spaces of continuous (analytic, differentiable) functions) with an orthogonal base and hence with the MAP (Theorem 3.1.(ii)) see [9, Section 2.5].

We now extend Theorem 3.1.(i) by proving that pseudoreflexivity is equivalent to having the $\lambda$-BAP for all $\lambda>1$.

THEOREM 3.2. $E$ is pseudoreflexive if and only if $E$ has $\lambda$-BAP for all $\lambda>1$.

Proof. The "only if" follows directly from Theorem 3.1. To prove the "if", let $x \in E, x \neq 0$, let $0<t<1$; we construct an $f \in E^{\prime} \backslash\{0\}$ with $|f(x)| \geq$ $t\|f\|\|x\|$. By assumption there is a $T \in F R(E)$ with $\|T\| \leq t^{-1 / 2}$ and $\|T(x)-x\|<\|x\|$. Then $\|T(x)\|=\|x\|$. Now $T(E)$, being finite-dimensional, is pseudoreflexive $\left([13,3.16(\right.$ iv) $)]$ ), so there is a $g \in(T(E))^{\prime}, g \neq 0$ such that $|g(T(x))| \geq t^{1 / 2}\|g\|\|T(x)\|$. Then $f:=g \circ T$ is in $E^{\prime} \backslash\{0\}$ and $\|f\| \leq$ $\|g\|\|T\| \leq t^{-1 / 2}\|g\|$. Thus, $|f(x)|=|g(T(x))| \geq t\|f\|\|T(x)\|=t\|f\|\|x\|$, and we are done.

In the real and complex theory, the $\lambda$-BAP for all $\lambda>1$ implies the MAP $([8,6 . \mathrm{III}])$, but not in our theory. In fact, it is shown in $[8,4.1]$ that, for non-spherically complete $K$, the Banach space $\ell^{\infty}$ of all bounded sequences in $K$ (with the supremum norm), has the $\lambda$-BAP for all $\lambda>1$ but does not have the MAP.

Now $\ell^{\infty}$ is not of countable type $([9,2.5 .15])$ and it was asked in $[8$, 4.5], whether spaces of countable type automatically had the MAP. The next example gives a negative answer to this question.

ExAmple 3.3. There exists a reflexive Banach space $E$ of countable type that does not have the MAP.

Proof. Let $K$ be not-spherically complete, let $K^{\vee}$ be its spherical completion. Then $K^{\vee}$ is in particular a $K$-Banach space.

We first prove that no pair of non-zero vectors in $K^{\vee}$ is an orthogonal system. In fact, let $x, y \in K^{\vee} \backslash\{0\}$. To show that $K x$ is not orthogonal to $K y$ we may assume that $|x|=|y|=1$ (as $\left|K^{\vee}\right|=|K|$ ). Now the residue class fields of $K^{\vee}$ and $K$ are isomorphic, so $\left|x y^{-1}-\mu\right|<1$ for some $\mu \in K$. It follows that $|x-\mu y|<1$, i.e. $K x$ is not orthogonal to $K y$.

Next we show that no infinite-dimensional subspace of $K^{\vee}$ has the MAP. In fact, suppose there is an infinite-dimensional subspace $G$ of $K^{\vee}$ with the MAP; we derive a contradiction. Let $x \in G \backslash\{0\}$. There is a $T \in F R(G)$ with $\|T\| \leq 1$ and $\|T(x)-x\|<\|x\|$. Then $\|T(x)\|=\|x\|$. For each $z \in \operatorname{Ker} T \backslash\{0\}$ 
we have $\|x-z\| \geq\|T(x)-T(z)\|=\|T(x)\|=\|x\|$, so $K x \perp \operatorname{Ker} T$, a contradiction with the assertion proved above.

Finally, $K^{\vee} / K$ is spherically complete $([13,4.2])$, so $\left(K^{\vee} / K\right)^{\prime}=\{0\}$ $([13,4.3])$. In particular, $K^{\vee}$ is not of countable type as a $K$-normed space $([13,3.16])$, so certainly admits closed infinite-dimensional subspaces $E$ of countable type (which are reflexive, $[13,4.18]$ ), finishing the construction.

Application. In [9, p. 95] the following problem was posed: Does there exist an absolutely convex edged set $C$ in some locally convex space $G$ over $K$ such that its closure $\bar{C}$ is not edged?

We shall use Example 3.3 to provide an affirmative answer when $K$ is not spherically complete and $G$ is even of countable type.

Let $\rho$ be the topology of pointwise convergence on $L(E)$, i.e. the Hausdorff locally convex topology on $L(E)$ defined by the family of seminorms $\left\{p_{x}: x \in\right.$ $E\}$, where $p_{x}(T):=\|T(x)\|, x \in E, T \in L(E)$. As usual, by pointwise convergence in $L(E)$ we mean $\rho$-convergence.

Then we have the following:

Let $K$ be not spherically complete. Let $E$ be a normed space of countable type without the $\operatorname{MAP}($ e.g. Example 3.3). Then $G:=(L(E), \rho)$ is a locally convex space of countable type and $C:=\{T \in F R(E):\|T\| \leq 1\}$ is an absolutely convex edged set in $L(E)$ such that $\bar{C}^{\rho}$ is not edged.

In fact, it suffices to prove that $(L(E), \rho)$ is of countable type; the rest follows from $[8,5.2]$. Observe that the map $(L(E), \rho) \rightarrow E^{E}, T \mapsto(T(x))_{x \in E}$ is a linear homeomorphism onto the image. Since $E$ is of countable type then, by the stability properties for locally convex spaces of countable type $([9,4.2 .13])$, we get that $(L(E), \rho)$ is of countable type.

We conclude this section by proving a stronger-looking, yet equivalent formulation of the MAP (Theorem 3.6). To this end we give two preparatory lemmas.

Lemma 3.4 (Extension lemma). Let $E$ be pseudoreflexive, let $D$ be a finite-dimensional subspace and let $0<\varepsilon_{1}<\varepsilon_{2}$. Then each $A \in L(D, E)$ with $\|A\| \leq \varepsilon_{1}$ can be extended to a $B \in F R(E)$ for which $\|B\| \leq \varepsilon_{2}$ and $B(E)=A(D)$.

Proof. By pseudoreflexivity, there is a projection $P$ of $E$ onto $D$ with $\|P\| \leq \varepsilon_{1}^{-1} \varepsilon_{2}$ (apply [13, 4.35] in the case when $K$ is spherically complete, and $[9,4.4 .6]$ for non-spherically complete $K)$. One verifies directly that $B:=A \circ P$ satisfies the requirements.

LEMMA 3.5 (Taking $\varepsilon=0$ in the definition of the MAP). Let $E$ have the MAP. Then for each finite set $X \subset E$ there is a $T \in F R(E)$ with $\|T\| \leq 1$ and $T(x)=x$ for all $x \in X$.

Proof. We may assume that $X \neq \emptyset$. The space $[X]$ is finite-dimensional, so it has (Theorem 2.1) a 1/2-orthogonal base $x_{1}, \ldots, x_{n}$. By scalar 
multiplication we can arrange that $\left\|x_{i}\right\| \geq 1$ for each $i$. By assumption there is a $T_{1} \in F R(E)$ with $\left\|T_{1}\right\| \leq 1$ and $\left\|T_{1}\left(x_{i}\right)-x_{i}\right\| \leq 1 / 4$ for each $i$. Now put $A:=\left(I_{E}-T_{1}\right) \mid[X]$. We next prove that $\|A\| \leq 1 / 2$. In fact, let $x \in[X]$, $x=\lambda_{1} x_{1}+\ldots+\lambda_{n} x_{n}$, where $\lambda_{i} \in K$. Then

$$
\begin{gathered}
\|A(x)\| \leq \max _{i}\left|\lambda_{i}\right|\left\|A\left(x_{i}\right)\right\|=\max _{i}\left|\lambda_{i}\right|\left\|x_{i}-T_{1}\left(x_{i}\right)\right\| \leq \frac{1}{4} \max _{i}\left|\lambda_{i}\right| \leq \\
\frac{1}{4} \max _{i}\left\|\lambda_{i} x_{i}\right\| \leq \frac{1}{4} 2\left\|\sum_{i} \lambda_{i} x_{i}\right\|=1 / 2\|x\|,
\end{gathered}
$$

and we are done.

$E$ is pseudoreflexive (Theorem 3.2), so by the extension lemma $3.4, A$ can be extended to a $B \in F R(E)$ with $\|B\| \leq 1$. Now put $T:=T_{1}+B$. We see that $T \in F R(E)$ and $T(x)=x$ for all $x \in X$. Finally, observe that $\|T\| \leq \max \left(\left\|T_{1}\right\|,\|B\|\right) \leq 1$, which completes the proof.

Now we arrive at the key result of this section.

THEOREM 3.6. Let E have the MAP. Then every finite-dimensional subspace is contained in a finite-dimensional orthocomplemented subspace.

Proof. Throughout the proof we fix a finite-dimensional subspace $D \neq$ $\{0\}$ and prove that $D$ is contained in a finite-dimensional orthocomplemented subspace, using a few steps.

(I) For a finite-dimensional subspace $F$ of $E$ and a $T \in F R(E)$ we say that $(F, T)$ is a proper pair if: (i) $T(E) \subset F$, (ii) $\|T\|=1$, (iii) $T(x)=x$ for all $x \in D$. (Notice that $D \subset F$ ).

Straightforward computation shows:

If $(F, T)$ is a proper pair then so is $\left(T(F), T^{2}\right)$.

(II) A proper pair $(F, T)$ is called minimal if there do not exist proper pairs $\left(F_{1}, T_{1}\right)$ with $\operatorname{dim} F_{1}<\operatorname{dim} F$.

By taking in Lemma 3.5 for $X$ a base of $D$, we obtain the existence of proper pairs. Then obviously:

There exist minimal proper pairs.

From now on in this proof we fix a minimal proper pair $(F, T)$; we will prove that $F$ is orthocomplemented (completing the proof of Theorem 3.6) as follows:

(III) $T(F)=F$. Proof. We have $T(E) \subset F$, so certainly $T(F) \subset F$. Now by $(\mathrm{I}),\left(T(F), T^{2}\right)$ is a proper pair, so by minimality $\operatorname{dim} T(F) \geq \operatorname{dim} F$, and we get (III).

(IV) $T \mid F$ is an isometry. Proof. Suppose not; we derive a contradiction. There is an $y \in F$ with $\|T(y)\| \neq\|y\|$. But, as $\|T\|=1$, we must have $\|T(y)\|<\|y\|$. We first prove that $K y \perp D$. For that it suffices to see that $\|y-x\| \geq\|x\|$ for all $x \in D$. This is clear if $\|y\| \neq\|x\|$, so suppose $\|y\|=\|x\|$ $(>\|T(y)\|)$. Then $\|y-x\| \geq\|T(y)-T(x)\|=\|T(y)-x\|=\|x\|$, and we are done. 
Next, consider the map $A: D+K y \mapsto K T(y)$ given by $A(x+\lambda y)=\lambda T(y)$ $(x \in D, \lambda \in K)$. Then from orthogonality (i.e. $\|x+\lambda y\| \geq\|\lambda y\|$ ) one arrives easily at $\|A\|=\|T(y)\| /\|y\|<1$. Since $E$ is pseudoreflexive (Theorem 3.2), by the extension lemma (Lemma 3.4) we can extend $A$ to a $B \in F R(E)$ with $\|B\|<1$ and $B(E)=A(D+K y)=K T(y)$.

Now define $U:=T-B$. From (i) $U(E) \subset T(E)+B(E) \subset F+K T(y) \subset F$, (ii) $\|U\|=\|T-B\|=\max (\|T\|,\|B\|)=1$, (iii) $U(x)=T(x)-B(x)=T(x)=$ $x$ for all $x \in D$, we infer that $(F, U)$ is a proper pair. Then, by $(\mathrm{I}),\left(U(F), U^{2}\right)$ is also a proper pair, so by minimality, $\operatorname{dim} U(F) \geq \operatorname{dim} F$. On the other hand, $U(y)=T(y)-B(y)=T(y)-A(y)=0$, so by finite-dimension considerations we have $\operatorname{dim} U(F)<\operatorname{dim} F$, a contradiction.

(V) $F$ is orthocomplemented. Proof. (i) $\operatorname{Ker} T \perp F$ : let $x \in \operatorname{Ker} T$, $y \in F$. To show $\|x-y\| \geq\|y\|$ we may assume $\|x\|=\|y\|$. Then, using (IV), we obtain $\|x-y\| \geq\|T(x)-T(y)\|=\|T(y)\|=\|y\|$.

(ii) $E=\operatorname{Ker} T+F$ : let $z \in E$. Then $T(z) \in F=T(F)$ by (III), so there is an $y \in F$ with $T(z)=T(y)$. Therefore, $z=(z-y)+y \in \operatorname{Ker} T+F$.

COROLlary 3.7. The following are equivalent.

( $\alpha$ ) E has the MAP.

( $\beta$ ) Each finite-dimensional subspace is contained in a finite-dimensional orthocomplemented subspace.

$(\gamma)$ There is a net $\left(P_{i}\right)_{i \in I}$ of finite rank orthoprojections $E \rightarrow E$ such that, for each $x \in E, P_{i}(x)=x$ for large $i$.

$(\delta)$ There is a net $\left(P_{i}\right)_{i \in I}$ of finite rank operators $E \rightarrow E$ with $\left\|P_{i}\right\| \leq 1$ for all $i$, such that $P_{i} \rightarrow I_{E}$ pointwise.

Proof. $(\alpha) \Longrightarrow(\beta)$ is Theorem 3.6, $(\gamma) \Longrightarrow(\delta)$ is obvious. For $(\beta)$ $\Longrightarrow(\gamma)$, let $I$ be the set of all finite-dimensional subspaces of $E$, directed by inclusion. By $(\beta)$ we can choose, for every $D \in I$, an orthoprojection $P_{D}$ of $E$ onto some finite-dimensional subspace $F \supset D$. Clearly $\left(P_{D}\right)_{D \in I}$ satisfies $(\gamma)$.

$(\delta) \Longrightarrow(\alpha)$. Let $\varepsilon>0$ and $X \subset E$ be finite. By $(\delta)$ there is a $j \in I$ such that $\left\|P_{j}(x)-x\right\| \leq \varepsilon$ for all $x \in X$, so $E$ has the MAP.

\section{Hereditary aspects of the MAP}

THEOREM 4.1. The MAP is stable for orthocomplemented subpaces.

Proof. Let $D$ be an orthocomplemented subspace of a normed space $E$ with the MAP. Let $\varepsilon>0$ and $X \subset D$ be finite. By assumption there is a $T_{1} \in F R(E)$ with $\left\|T_{1}\right\| \leq 1$ and $\left\|T_{1}(x)-x\right\| \leq \varepsilon$ for all $x \in X$. Now let $P$ be an orthoprojection of $E$ onto $D$ and put $T:=\left(P \circ T_{1}\right) \mid D$. Then clearly $T \in F R(D)$ and $\|T\| \leq 1$. Also, for each $x \in X,\|T(x)-x\|=$ $\left\|\left(P \circ T_{1}\right)(x)-P(x)\right\| \leq\|P\|\left\|T_{1}(x)-x\right\| \leq \varepsilon$. Hence $D$ has the MAP. 
To describe the stability of the MAP for dense subspaces we need a general lemma.

Lemma 4.2. Let $D$ be a finite-dimensional subspace of $E$, let $F$ be a dense subspace of $E$. Then, for each $\varepsilon>0$, there is a $T \in L(D, F)$ with $\|x-T(x)\| \leq \varepsilon\|x\|$ for all $x \in D$.

Proof. Let $x_{1}, \ldots, x_{n}$ be a $1 / 2$-orthogonal base of $D$ (Theorem 2.1). By density there are $y_{1}, \ldots, y_{n} \in F$ such that $\left\|x_{i}-y_{i}\right\| \leq(\varepsilon / 2)\left\|x_{i}\right\|$ for all $i$. Define $T: D \rightarrow F$ by $T\left(x_{i}\right):=y_{i}(i \in\{1, \ldots, n\})$ and linearity. Then $T \in L(D, F)$. To get the conclusion, let $x=\sum_{i} \lambda_{i} x_{i} \in D$. Then we have $\|x-T(x)\|=\left\|\sum_{i} \lambda_{i}\left(x_{i}-y_{i}\right)\right\| \leq \max _{i}\left|\lambda_{i}\right|\left\|x_{i}-y_{i}\right\| \leq(\varepsilon / 2) \max _{i}\left|\lambda_{i}\right|\left\|x_{i}\right\|$, which by $1 / 2$-orthogonality, is $\leq \varepsilon\|x\|$, completing the proof.

TheOREM 4.3. (Stability of the MAP for dense subspaces and closures) Let $E_{1}$ be a dense subspace of a normed space $E_{2}$. Then $E_{1}$ has the MAP if and only if $E_{2}$ has the MAP. In particular, the completion of a normed space with the MAP has the MAP.

Proof. (i) Suppose $E_{1}$ has the MAP. To prove that $E_{2}$ has the MAP, let $\varepsilon>0$ and $X:=\left\{x_{1}, \ldots, x_{n}\right\} \subset E_{2}$; we construct a $T_{2} \in F R\left(E_{2}\right)$ with $\left\|T_{2}\right\| \leq 1$ and $\left\|T_{2}\left(x_{i}\right)-x_{i}\right\| \leq \varepsilon$ for all $i$. By density there are $y_{1}, \ldots, y_{n} \in E_{1}$ such that $\left\|x_{i}-y_{i}\right\| \leq \varepsilon$ for each $i$. By assumption there is a $T_{1} \in F R\left(E_{1}\right)$ with $\left\|T_{1}\right\| \leq 1$ and $\left\|T_{1}\left(y_{i}\right)-y_{i}\right\| \leq \varepsilon$ for each $i$. $T_{1}$ extends uniquely to a $T_{2} \in L\left(E_{2}\right)$. As $T_{1}\left(E_{1}\right)$ is finite-dimensional, hence complete, we have $T_{2}\left(E_{2}\right) \subset \overline{T_{1}\left(E_{1}\right)}=T_{1}\left(E_{1}\right)$, so that $T_{2} \in F R\left(E_{2}\right)$. Clearly $\left\|T_{2}\right\| \leq 1$. Finally, $\left\|T_{2}\left(x_{i}\right)-x_{i}\right\|=\left\|\left(T_{2}\left(x_{i}\right)-T_{2}\left(y_{i}\right)\right)+\left(T_{2}\left(y_{i}\right)-y_{i}\right)+\left(y_{i}-x_{i}\right)\right\| \leq \max \left(\| x_{i}-\right.$ $\left.y_{i}\|,\| T_{1}\left(y_{i}\right)-y_{i}\|,\| y_{i}-x_{i} \|\right) \leq \varepsilon$ for each $i$, showing that $E_{2}$ has the MAP.

(ii) Suppose $E_{2}$ has the MAP. To prove that $E_{1}$ has the MAP, let $\varepsilon>0$ and $\emptyset \neq X \subset E_{1}$ be finite. By assumption there is a $T_{2} \in F R\left(E_{2}\right)$ with $\left\|T_{2}\right\| \leq 1$ and $\left\|T_{2}(x)-x\right\| \leq \varepsilon$ for all $x \in X$. Now let $\delta \in(0,1)$ with $\delta \max \left\{\left\|T_{2}(x)\right\|: x \in X\right\} \leq \varepsilon$. By Lemma 4.2, there is a $S \in L\left(T_{2}\left(E_{2}\right), E_{1}\right)$ such that $\|z-S(z)\| \leq \delta\|z\|$ for all $z \in T_{2}\left(E_{2}\right)$. Finally, put $T_{1}:=\left(S \circ T_{2}\right) \mid E_{1}$. Then $T_{1} \in F R\left(E_{1}\right)$ and $\left\|T_{1}\right\| \leq 1$ (as $S$ is an isometry). Also, for each $x \in X$,

$$
\begin{aligned}
& \left\|T_{1}(x)-x\right\|=\left\|\left(\left(S \circ T_{2}\right)(x)-T_{2}(x)\right)+\left(T_{2}(x)-x\right)\right\| \leq \\
& \max \left(\left\|S\left(T_{2}(x)\right)-T_{2}(x)\right\|,\left\|T_{2}(x)-x\right\|\right) \leq \max \left(\delta\left\|T_{2}(x)\right\|, \varepsilon\right) \leq \varepsilon,
\end{aligned}
$$

proving that $E_{1}$ has the MAP.

As a next step we consider algebraic orthogonal direct sums.

THEOREM 4.4. Let $\left(E_{i}\right)_{i \in I}$ be a collection of normed spaces. Then its algebraic orthogonal direct sum $\bigoplus_{i}^{a} E_{i}$ has the MAP if and only if each $E_{i}$ has the MAP. 
Proof. Each $E_{i}$ is orthocomplemented in $E:=\bigoplus_{i}^{a} E_{i}$ (we identify each $E_{i}$ with its image under the natural injection $E_{i} \rightarrow E$ ). Thus, if $E$ has the MAP then so has each $E_{i}$ (Theorem 4.1).

Now assume that each $E_{i}$ has the MAP. For each $i$, let $P_{i}$ be the canonical orthoprojection $E \rightarrow E_{i}, x=\left(x_{i}\right)_{i \in I} \mapsto x_{i}$. Let $\varepsilon>0$ and $X \subset E$ be finite. There is a finite set $J \subset I$ for which $X \subset \sum_{j \in J} P_{j}\left(E_{j}\right)$. Then $X \subset$ $\sum_{j \in J} P_{j}(X)$. By assumption there is, for each $j \in J$, a $T_{j} \in F R\left(E_{j}\right)$ with $\left\|T_{j}\right\| \leq 1$ and $\left\|T_{j}(z)-z\right\| \leq \varepsilon$ for all $z \in P_{j}(X)$. Now define $T: E \rightarrow E$ by the formula $(T(x))_{i}=T_{i}\left(P_{i}(x)\right)$ if $i \in J ;(T(x))_{i}=0$ otherwise. Then $T \in$ $F R(E),\|T\| \leq 1$ and, for each $x \in X$, we have $\|T(x)-x\|=\max _{i \in J} \|(T(x))_{i}-$ $x_{i}\left\|=\max _{i \in J}\right\| T_{i}\left(x_{i}\right)-x_{i} \| \leq \varepsilon$, and we are done.

The step towards orthogonal direct sums is now easy:

Corollary 4.5. Let $\left(E_{i}\right)_{i \in I}$ be a collection of normed spaces. Then $\bigoplus_{i} E_{i}$ has the MAP if and only if each $E_{i}$ has the MAP.

Proof. Combine Theorem 4.3 and Theorem 4.4.

As finite-dimensional spaces trivially have the MAP, the next result follows directly.

Corollary 4.6. A Banach space with the FDDP has the MAP.

The converse of Corollary 4.6 does not hold.

ExAMPLE 4.7. There exists a Banach space $E$ having the MAP but not the FDDP.

Proof. In $[7,3.6]$, for non-spherically complete $K$, a closed subspace $E$ of $\ell^{\infty}$ was constructed that has no orthogonal base but whose finite-dimensional subspaces are orthocomplemented. Then certainly $E$ has the MAP (e.g. Corollary 3.7) and it is also easily seen that finite-dimensional subspaces of $E$ have orthogonal bases. Then $E$, having no orthogonal base, cannot have the FDDP.

However, for spaces of countable type we do have a converse.

LEMMA 4.8. Let $E$ be a normed space of countable type having the MAP. Then there exists an orthogonal sequence $\left(D_{n}\right)_{n \in \mathbb{N}}$ of finite-dimensional subspaces such that $\sum_{n} D_{n}$ is dense in $E$.

Proof. Let $x_{1}, x_{2}, \ldots \in E$ be such that $\left[x_{1}, x_{2}, \ldots\right]$ is dense in $E$. We will construct inductively an orthogonal sequence $D_{1}, D_{2}, \ldots$ of finite-dimensional subspaces, and subspaces $H_{1}, H_{2}, \ldots$ such that, for each $n,(\mathrm{i})\left[x_{1}, \ldots, x_{n}\right] \subset$ $D_{1}+\ldots+D_{n}$, (ii) $H_{n}$ is an orthocomplement of $D_{1}+\ldots+D_{n}$. (This will prove the lemma). To this end, we first apply Corollary 3.7 to conclude that $K x_{1}$ is contained in an orthocomplemented finite-dimensional subspace, say, $D_{1}$. Let 
$H_{1}$ be an orthocomplement of $D_{1}$. For the step $n \rightarrow n+1$, suppose we have constructed $D_{1}, \ldots, D_{n}$ and $H_{1}, \ldots, H_{n}$ in the above fashion. Then $x_{n+1}$ has a unique decomposition $x_{n+1}=y_{n}+h_{n}$, where $y_{n} \in D_{1}+\ldots+D_{n}, h_{n} \in H_{n}$. Now by Theorem $4.1 H_{n}$ has the MAP, so $h_{n}$ lies in a finite-dimensional subspace $D_{n+1}$ of $H_{n}$ that is orthocomplemented in $H_{n}$. Let $H_{n+1}$ be such an orthocomplement. Then $H_{n+1}$ is trivially an orthocomplement of $D_{1}+$ $\ldots+D_{n+1}$ in $E$ and $x_{n+1}=y_{n}+h_{n} \in D_{1}+\ldots+D_{n}+D_{n+1}$, which proves the step $n \rightarrow n+1$.

We can now formulate the following result.

THEOREM 4.9. A Banach space of countable type has the MAP if and only if it has the FDDP.

REMARK 4.10. Throughout this remark, let $K$ be not spherically complete. Let $E$ have the MAP and let $D$ be a subspace of $E$.

1. (Subspaces) Does $D$ have the MAP?

We know that the answer is yes if $D$ is finite-dimensional, or orthocomplemented (Theorem 4.1) or dense (Theorem 4.3), but the general question remains open. Notice that, for Banach spaces $E$ of countable type, the above question is by Theorem 4.9 equivalent to:

Let $E$ have the FDDP. Do subspaces have the FDDP?

Observe that the related problem: Let $E$ have an orthogonal base. Do subspaces have an orthogonal base?, is solved affirmatively $\left(\left[\begin{array}{ll}9,2.3 .22\end{array}\right]\right)$.

2. (Quotients) Let $D$ be closed. Does $E / D$ have the MAP?

The answer is "no" in general: it suffices to take a Banach space $F$ without the MAP and observe that, thanks to [9, 2.5.6], $F$ is a quotient of some Banach space with an orthogonal base (which has the MAP by Theorem 3.1(ii)). If we choose for $F$ a space of countable type (see Example 3.3) we can even conclude by $[9,2.3 .28]$ that $F$ is a quotient of $c_{0}$.

However, quotients of $E$ by finite-dimensional subspaces have the MAP, as we show in the next result.

THEOREM 4.11. Let $E$ have the MAP, let $D$ be a finite-dimensional subspace. Then $E / D$ has the MAP.

Proof. Let $M$ be a finite-dimensional subspace of $E / D$. We construct (Lemma 3.5) a $S \in F R(E / D)$ with $\|S\| \leq 1$ and $S(z)=z$ for all $z \in M$. Let $\pi: E \rightarrow E / D$ be the canonical quotient map. Then $\pi^{-1}(M)$ is finitedimensional, so by assumption and Lemma 3.5, there is a $T \in F R(E)$ with $\|T\| \leq 1$ and $T(x)=x$ for all $x \in \pi^{-1}(M)$, in particular, $T(x)=x$ for all $x \in \bar{D}$, as $D \subset \pi^{-1}(M)$. Now, let $S: E / D \rightarrow E / D$ be the map given by $S(\pi(x))=\pi(T(x))(x \in E)$. Then $S$ is a well-defined finite rank operator with $\|S\| \leq 1$. Also, for each $z \in M$ there is an $x \in \pi^{-1}(M)$ for which $\pi(x)=z$. Thus, $S(z)=S(\pi(x))=\pi(T(x))=\pi(x)=z$, so $S$ meets the requirements. 
We conclude this section with the following for tensor products.

ThEOREM 4.12. Let E, $F$ have the MAP. Then the tensor product $E \otimes F$ and its completion $E \hat{\otimes}_{\pi} F$ have the MAP.

Proof. By Theorem 4.3 we only need to consider $E \otimes F$. Let $\varepsilon>0$ and $\{0\} \neq Z \subset E \otimes F$ be finite. There are non-empty finite sets $\{0\} \neq X \subset E$, $\{0\} \neq Y \subset F$ such that every $z \in Z$ can be written as a finite sum, $z=\sum_{i} x_{i} \otimes$ $y_{i}, x_{i} \in X, y_{i} \in Y$. Let $M_{X}:=\max \{\|x\|: x \in X\}, M_{Y}:=\max \{\|y\|: y \in Y\}$. By assumption there exist $T \in F R(E), S \in F R(F)$ with $\|T\| \leq 1,\|S\| \leq 1$ and

$$
\|T(x)-x\| \leq \frac{\varepsilon}{M_{Y}} \text { for all } x \in X, \quad\|S(y)-y\| \leq \frac{\varepsilon}{M_{X}} \text { for all } y \in Y .
$$

Then $T \otimes S \in F R(E \otimes F)$ and $\|T \otimes S\| \leq 1$. Now, for each $x \in X, y \in Y$,

$$
\begin{aligned}
& \|(T \otimes S)(x \otimes y)-x \otimes y\|=\|T(x) \otimes S(y)-T(x) \otimes y+T(x) \otimes y-x \otimes y\| \\
& \leq \max (\|T(x)\|\|S(y)-y\|,\|T(x)-x\|\|y\|) \leq \max \left(M_{X} \frac{\varepsilon}{M_{X}}, \frac{\varepsilon}{M_{Y}} M_{Y}\right)=\varepsilon .
\end{aligned}
$$

Then it is easily seen that $\|(T \otimes S)(z)-z\| \leq \varepsilon$ for all $z \in Z$, and we are done.

Problem Let $E \otimes F$ have the MAP, and suppose $E \neq\{0\}, F \neq\{0\}$. Does it follow that $E$ and $F$ have the MAP?

\section{Comparison with the Classical Case}

Finally we compare the results given in this paper with their classical (or archimedean) counterparts, for Banach spaces over $\mathbb{R}$ or $\mathbb{C}$.

Since every space over a spherically complete $K$ has the MAP (Theorem 3.1(ii)), in this section we assume that $E$ is a non-archimedean Banach space over a non-spherically complete $K$. Also, we assume that $\mathcal{E}$ is a Banach space over $\mathbb{R}$ or $\mathbb{C}$.

The notion of the MAP for $\mathcal{E}$ is just a translation of the one given in Definition 1.1.

I. The classical approximation theory was initiated in the Grothendieck's memoir [5], where among other things, he studied the MAP. At that moment all known classical Banach spaces had the MAP. He conjectured that every space $\mathcal{E}$ had this property. It was not until 1973, when Enflo proved in [3] that the conjecture of Grothendieck was false. He gave an example of a separable reflexive space $\mathcal{E}$ without the MAP. For more examples of classical Banach spaces with and without the MAP see e.g. [2] and its references on the subject.

In the non-archimedean setting, the space $E$ of Example 3.3 plays the role of the classical example given by Enflo: it is a reflexive Banach space of countable type for which the non-archimedean version of the conjecture of Grothendieck is false. 
II. In the classical case one verifies:

(i) $([2,3.10]) c_{0}$ has the MAP in every equivalent norm.

(ii) ([4, VI.3]) There exists a closed subspace $\mathcal{E}$ of $c_{0}$ such that $\mathcal{E}$ has the 8-BAP but fails the MAP.

The non-archimedean counterparts of these classical results are false.

To see that (i) is false, let $E$ be the Banach space of countable type, without the MAP, constructed in Example 3.3. Then $E$ is linearly homeomorphic to $c_{0}([9,2.3 .9])$, i.e. there is an equivalent norm $\|$. $\|$ on $c_{0}$, such that $\left(c_{0},\|\|.\right)$ is isometrically isomorphic to $E$, so $\left(c_{0},\|\cdot\|\right)$ does not have the MAP.

Falsity of (ii) follows from the fact that every closed subspace of $c_{0}$ has an orthogonal base (Remark 4.10.1), so it has the MAP (Theorem 3.1(ii)).

III. In the archimedean theory we have $([2,3.6])$ : If $\mathcal{E}$ is a separable dual space such that

(*) for every $\varepsilon>0$ and every compact set $X \subset \mathcal{E}$ there exists a $T \in F R(\mathcal{E})$ with $\|T(x)-x\| \leq \varepsilon$ for all $x \in X$,

then $\mathcal{E}$ has the MAP.

The non-archimedean counterpart of this classical result is false.

Indeed, let $E$ be the reflexive (hence dual) space of countable type of Example 3.3. We know that $E$ does not have the MAP. Let us see that $E$ satisfies $(*)$, and we are done. $E$ is reflexive, hence pseudoreflexive, i.e. $E$ has the $\lambda$-BAP for all $\lambda>1$ (Theorem 3.2). Now, as the finite sets in Definition 1.1 can be replaced by compact sets $([8,3.2])$, we derive that $E$ satisfies $\left(^{*}\right)$.

IV. Let us discuss the situation in III when we consider the approximation properties (in the archimedean and in the non-archimedean case) obtained from the MAP and $\left(^{*}\right)$, by imposing the operator $T$ appearing in their definitions to be compact, instead of finite rank. Let us call CMAP and $\left(\mathrm{C}^{*}\right)$, respectively, the approximation properties obtained after these replacements. Then, an open problem in the classical theory $([2,8.7])$ is the following:

$\mathcal{E}$ is a separable dual space with property $\left(\mathrm{C}^{*}\right) \Longrightarrow \mathcal{E}$ has the $\mathrm{CMAP}$ ?

In the non-archimedean case the answer to this problem is NO.

Indeed, it was proved in $[8,3.2]$ that $E$ has the MAP if and only if $E$ has the CMAP. Then the non-archimedean result given in III provides the desired negative answer.

V. It is well-known (see e.g. [5, I.5.39]) that $\mathcal{E}$ has the MAP if and only if it has an approximating net, i.e. a net $\left(P_{i}\right)_{i \in I}$ of finite rank operators $\mathcal{E} \rightarrow \mathcal{E}$ with $\left\|P_{i}\right\| \leq 1$ for all $i$, such that $P_{i} \rightarrow I_{\mathcal{E}}$ pointwise (this result is the archimedean version of $(\alpha) \Longleftrightarrow(\delta)$ of Corollary 3.7$)$. But there exist spaces $\mathcal{E}$ with the MAP and:

(i) having no approximating nets consisting of finite rank projections,

(ii) having no finite-dimensional decompositions $([2,6.1])$. 
In fact, it is proved in $[2,5.2]$ (and the comments before it) that there is a separable reflexive space $\mathcal{E}$ with property $(*)$, hence with the MAP (see III), for which there are not bounded nets of finite rank projections $\mathcal{E} \rightarrow \mathcal{E}$ converging pointwise to $I_{\mathcal{E}}$. The non-existence of such bounded nets implies (i) and (ii).

The assertions (i) and (ii) above show, respectively, that the classical counterparts of $(\alpha) \Longrightarrow(\gamma)$ of Corollary 3.7 and of the "only if" of Theorem 4.9 are false.

ACKNOWLEDGEMENTs.

Research of the first author partially supported by Ministerio de Ciencia e Innovación, MTM2010-20190-C02-02.

\section{REFERENCES}

[1] G. Bachman, Introduction to $p$-Adic Numbers and Valuation Theory, Academic Press, New York, 1964.

[2] P. G. Casazza, Approximation properties, Handbook of the geometry of Banach spaces, Vol. I, North-Holland, Amsterdam (2001), 271-316.

[3] P. Enflo, A counterexample to the approximation problem in Banach spaces, Acta Math. 130 (1973), 309-317.

[4] G. Godefroy, The Banach space $c_{0}$, Extracta Math. 16 (2001), 1-25.

[5] A. Grothendieck, Produits tensoriels topologiques et espaces nucléaires, Mem. Amer. Math. Soc. 16 (1955), 1-140.

[6] A. Kubzdela, On finite-dimensional normed spaces over $\mathbb{C}_{p}$, Contemp. Math. 384 (2005), 169-185

[7] A. Kubzdela, On non-Archimedean Hilbertian spaces, Indag. Math. (N.S.) 19 (2008), 601-610.

[8] C. Perez-Garcia, Bounded approximation properties in non-archimedean Banach spaces, Math. Nach. 285 (2012), 1255-1263.

[9] C. Perez-Garcia and W. H. Schikhof, Locally Convex Spaces over Non-Archimedean Valued Fields, Cambridge University Press, Cambridge, 2010.

[10] A. M. Robert, A Course in p-Adic Analysis, Springer, Berlin, 2000.

[11] W. H. Schikhof, Ultrametric Calculus. An Introduction to $p$-Adic Analysis, Cambridge University Press, Cambridge, 1984.

[12] W. H. Schikhof, Banach spaces over nonarchimedean valued fields, Topology Proc. 24 (1999), 547-581.

[13] A. C. M. van Rooij, Non-Archimedean Functional Analysis, Dekker, New York, 1978.

C. Perez-Garcia

Department of Mathematics, Facultad de Ciencias

Universidad de Cantabria

Avda. de los Castros s/n, 39071, Santander

Spain

E-mail: perezmc@unican.es

W. H. Schikhof

Weezenhof 3607

6536 HC Nijmegen

The Netherlands

E-mail: schikhof@upcmail.nl

Received: 30.12.2013. 